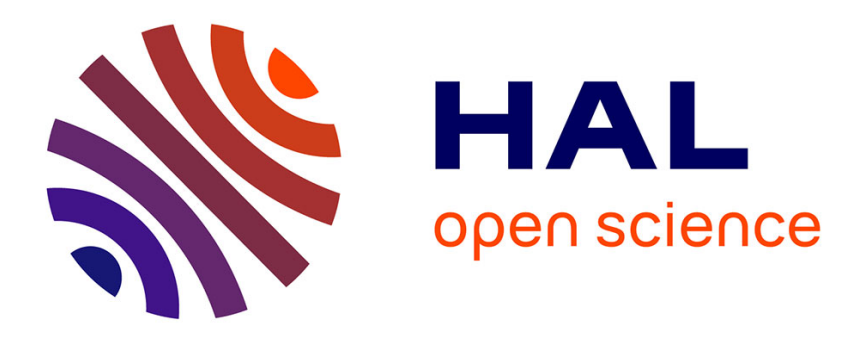

\title{
1 enfant sur 30 conçu par assistance médicale à la procréation en France
}

Elise de La Rochebrochard

\section{To cite this version:}

Elise de La Rochebrochard. 1 enfant sur 30 conçu par assistance médicale à la procréation en France. Population et sociétés, 2018, 556, 10.3917/popsoc.556.0001 . hal-01990066

\section{HAL Id: hal-01990066 https://hal.science/hal-01990066}

Submitted on 22 Jan 2019

HAL is a multi-disciplinary open access archive for the deposit and dissemination of scientific research documents, whether they are published or not. The documents may come from teaching and research institutions in France or abroad, or from public or private research centers.
L'archive ouverte pluridisciplinaire HAL, est destinée au dépôt et à la diffusion de documents scientifiques de niveau recherche, publiés ou non, émanant des établissements d'enseignement et de recherche français ou étrangers, des laboratoires publics ou privés. 


\title{
Population \& Sociétés
}

\section{1 enfant sur 30 conçu par assistance médicale à la procréation en France}

\author{
Élise de La Rochebrochard*
}

\begin{abstract}
L'assistance médicale à la procréation (AMP) s'est beaucoup développée depuis la naissance du premier « bébé-éprouvette » au monde il y a 40 ans. Elise de La Rochebrochard en fait un bilan pour la France. Elle nous explique combien d'enfants conçus par AMP naissent dans le pays chaque année, détaille notamment les méthodes utilisées et la proportion d'enfants issus d'un don de gamètes, et estime le nombre total de naissances en France dues à l'AMP depuis ses débuts.
\end{abstract}

Le premier «bébé-éprouvette » au monde, Louise Brown, fête ses 40 ans le 25 juillet 2018. La naissance de cette anglaise signe une véritable révolution dans l'assistance médicale à la procréation (AMP) (encadré 1). Cette technique permet en effet la fécondation d'un ovule par un spermatozoïde hors du corps de la femme, dans «l'éprouvette " du biologiste, in vitro selon le terme technique. Jusqu'à cette première fécondation in vitro (FIV), les techniques d'AMP se cantonnaient à des inséminations " artificielles » (IA) qui consistent à déposer les spermatozoïdes du conjoint (IAC) ou d'un donneur (IAD) au niveau du col de l'utérus ou dans la cavité utérine pour qu'ils aillent féconder naturellement un ovocyte dans le corps de la femme ${ }^{(1)}$.

\section{L'AMP est à l'origine d'une naissance sur trente}

En France, en 2018, 1 enfant sur 30 (3,4\%) devrait être conçu grâce à une technique d'AMP, qu'il s'agisse d'une FIV ou d'une IA (figure 1 et encadré 2 ). Cependant la FIV domine désormais le paysage de l'assistance médicale, représentant $70 \%$ des enfants conçus par AMP.

* Ined - Inserm - Université Paris-Sud - Université de Versailles-SaintQuentin

(1) La prise en charge médicale de l'infécondité débute souvent avec un traitement par stimulation hormonale simple (c'est-à-dire sans insémination artificielle ni fécondation in vitro). Ces traitements ne sont pas inclus dans l'AMP car il n'y a pas de manipulation des spermatozoïdes, de l'ovocyte ou de l'embryon. II n'existe aucun suivi de la fréquence de recours à ces traitements de stimulations hormonales simples ni du nombre d'enfants conçus.
Premier «bébé éprouvette " français, Amandine est conçue en 1981 et naît le 24 février 1982. Par la suite le nombre de FIV ne va cesser de croitre selon un rythme remarquablement linéaire (figure 1). La proportion d'enfants conçus par FIV progresse de $+0,5 \%$ tous les 7 à 8 ans. En 2011, 2,0 \% des enfants étaient conçus par FIV, et si la tendance se poursuit, la proportion atteindra

Figure 1. Évolution de la proportion d'enfants conçus par assistance médicale à la procréation

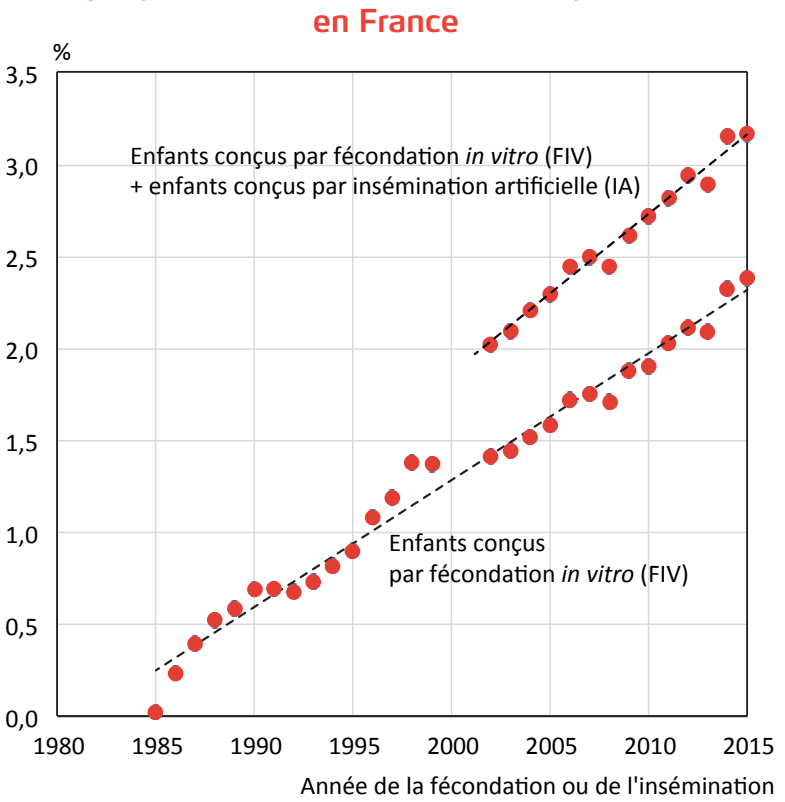

Élise de La Rochebrochard, Population \& Sociétés n556, INED, juin 2018. 
Encadré 1. Glossaire des sigles

AMP : Assistance médicale à la procréation

CECOS : Centre d'étude et de conservation des œufs et du sperme humains

DPI : Diagnostic pré-implantatoire

FIV : Fécondation in vitro

GPA : Gestation pour autrui

IA : Insémination artificielle

IAC: Insémination artificielle avec spermatozoïdes du conjoint

IAD : Insémination artificielle avec spermatozoïdes de donneur

ICSI : Injection intra-cytoplasmique de spermatozoïde

(traduction de l'anglais : Intra Cytoplasmic Sperm Injection)

TEC: Transfertd'embryonscongelés
Figure 2. Nombre cumulé d'enfants conçus par fécondation in vitro (FIV) en France depuis 1981

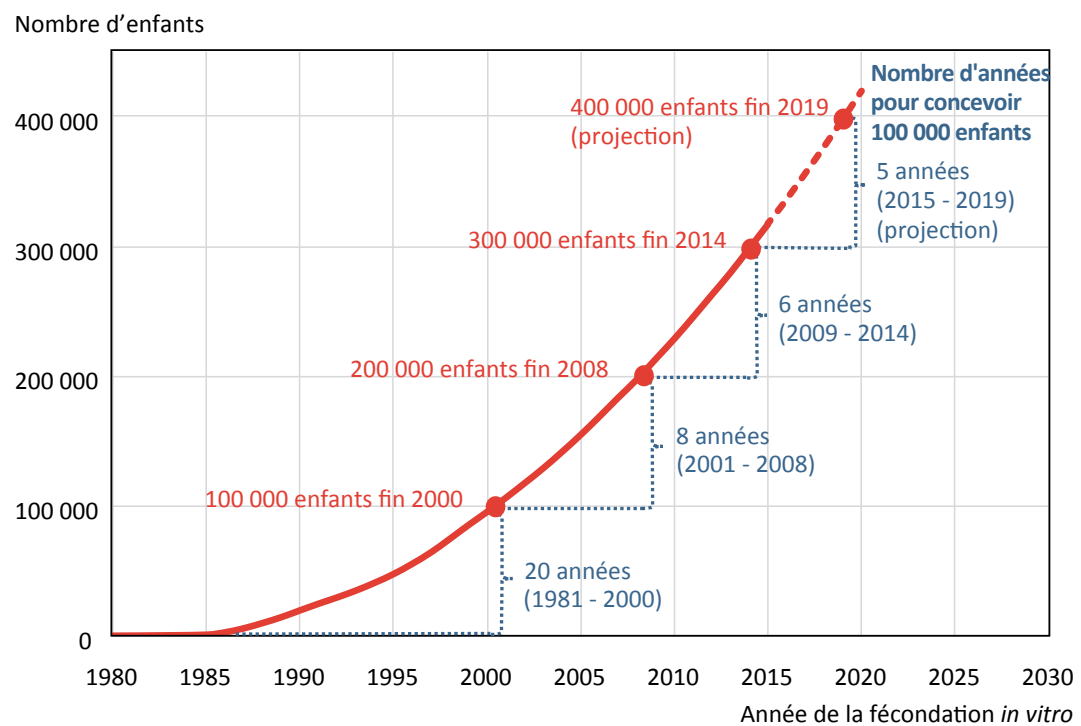

Élise de La Rochebrochard, Population \& Sociétés n 556, INED, juin 2018.
2,5\% en 2018, soit 1 enfant sur 40 (plus de 20000 enfants). Le recours croissant à la FIV a été soutenu par les progrès technologiques qui lui permettent désormais de répondre aussi bien aux infertilités d'origine féminine qu'à celles d'origine masculine. Initialement la FIV n'avait été conçue que pour répondre aux infertilités féminines d’origine tubaire (trompes altérées ou bouchées). Mais depuis 1992, une nouvelle technique de FIV permet aussi de prendre en charge les infertilités masculines : l'injection intra-cytoplasmique de spermatozoïde (ICSI). Elle consiste à sélectionner un spermatozoïde qui est directement introduit dans l'ovocyte. L'ICSI a connu un développement extrêmement important puisqu'en 20122015, deux fécondations in vitro sur trois réalisées en France le sont avec cette méthode.

\section{Bientôt 400000 enfants conçus par FIV en France}

Les FIV réalisées durant les deux premières décennies d'activité (1981-2000) ont permis la naissance de 100000 enfants au total en France (figure 2). Le mouvement s'amplifie ensuite puisque l'effectif de 200000 enfants est atteint fin 2008 et celui de 300000 fin 2014. Si la tendance se poursuit au rythme observé depuis 30 ans, la France devrait arriver d'ici la fin 2019 à un total de 400000 enfants conçus par FIV. Cette dynamique s'observe également dans le reste du monde. Ainsi, le Comité international de surveillance de l'AMP (ICMART) estimait en 2013 que 5 millions d'enfants avaient été conçus par FIV dans le monde au total, dont la moitié au cours des 6 dernières années [3].

Les enfants conçus par FIV comportent une proportion importante de naissances multiples : en France, dans les années 1990, il ne naissait pas moins de 130 enfants pour
100 accouchements obtenus suite à une FIV. Au début de la FIV, pour augmenter les chances d'obtenir une grossesse, les médecins transféraient beaucoup d'embryons, souvent 4 ou plus à la fois (39\% des cas en France en 1988). Les naissances multiples augmentent cependant fortement les risques pour la santé des enfants. Les médecins en ont pris peu à peu conscience et ont transféré moins d'embryons pour réduire le risque de naissances multiples. Ils ont ainsi privilégié le transfert de 3 embryons (environ $40 \%$ des cas en 1997) puis de 2 embryons (environ $60 \%$ des cas en 2009). Il n'y a donc plus actuellement que 110 enfants pour 100 accouchements suite à une FIV, mais c'est une fréquence qui reste beaucoup plus élevée que dans le cas d'une grossesse « naturelle » (101 enfants pour 100 accouchements). Aujourd'hui, l'objectif est de réduire encore la fréquence des naissances gémellaires en favorisant le transfert d'un seul embryon, les autres étant le cas échéant congelés et transférés lors de tentatives ultérieures. En France, plus de $40 \%$ des FIV réalisées en 2015 ont donné lieu à un transfert unique d'embryon et cette proportion devrait augmenter dans les prochaines années. La Finlande et la Suède ouvrent la voie avec une politique très volontariste ayant permis d'atteindre près de $80 \%$ de transferts d'un seul embryon.

\section{$5 \%$ seulement des enfants conçus par AMP le sont avec tiers donneur}

Dans l'imaginaire collectif, l'AMP est souvent associée à l'idée du recours à un tiers pour avoir un enfant, que ce soit via un don de spermatozoïdes, d'ovules, d'embryons (lorsque ceux d'un couple sont accueillis par un autre couple), ou en faisant appel à une gestatrice pour autrui (GPA). En réalité, la quasi-totalité des enfants conçus par AMP en France (95 \% en 2015) le sont avec les gamètes de 
leurs deux parents. L'AMP avec tiers donneur concerne très largement le don de spermatozoïdes $(4 \%$ des naissances AMP, soit environ 1000 enfants par an) et de manière marginale le don d'ovocytes ( $1 \%$ des naissances AMP, soit environ 250 enfants par an). L'accueil d'embryons est statistiquement négligeable ( $0,01 \%$ des naissances AMP, soit environ 25 à 30 enfants chaque année), tandis que la gestation pour autrui est interdite en France.

Le don de gamètes est gratuit et anonyme en France. Il est régi par les $\mathrm{CECOS}^{(2)}$, connus dans le grand public sous le terme de banques de sperme et d'ovocytes. La pénurie de donneurs en France ne permet pas de répondre à la demande des couples hétérosexuels infertiles en âge reproductif, en particulier la demande d'ovocytes. La loi française restreint pour l'instant l'accès à l'AMP à ces seuls couples, mais un élargissement de cet accès est souhaité par une partie de la société. En l'état actuel de la législation, les " exclus » de l'AMP regroupent les femmes ayant fêté leur $43^{\mathrm{e}}$ anniversaire et demandant un don d'ovocytes, car elles sont touchées par le vieillissement ovocytaire qui s'accélère dès 37 ans, les femmes seules ou les couples de femmes demandant un don de spermatozoïdes, les couples d'hommes ayant besoin d'un don d'ovocytes et d'une gestatrice, et les femmes ne pouvant mener une grossesse et demandant une GPA.

Les Français qui ne peuvent accéder à l'AMP avec tiers donneur peuvent décider de recourir à une AMP dite transnationale pour concevoir l'enfant désiré au-delà des frontières nationales, souvent au sein même de l'Europe [4]. Les couples hétérosexuels en âge reproductif peuvent demander le remboursement de cette AMP transnationale au CNSE (centre de remboursement de soins à l'étranger) qui a reçu 1499 demandes de ce type en 2015. Cependant, ces recours sont souvent perçus comme illégaux et il est probable que tous ne font pas valoir leurs droits. Par ailleurs, les « exclus » de l'AMP ne peuvent pas bénéficier de cette prise en charge par le CNSE.

\section{L'AMP avec tiers donneur dans l'Union européenne : un paysage déséquilibré}

Environ $10 \%$ des FIV réalisées dans l'Union européenne (UE) ont lieu en France d'après le bilan européen d'activité d'AMP pour l'année 2013 (encadré 2). Cette proportion est moindre que la part de la France dans les naissances de l'UE (15\%). En comparaison, l'Allemagne réalise $13 \%$ des FIV au sein de l'UE, une proportion identique à sa contribution aux naissances $(13 \%)$, tandis que l'Italie réalise $12 \%$ des FIV alors qu'elle ne contribue aux naissances qu'à hauteur de $10 \%$. Malgré ces variations entre pays, l'activité FIV des pays est globalement cohérente avec leur poids démographique: les cinq grands pays de l'UE (Royaume-Uni, France, Allemagne, Italie et Espagne) contribuent pour $63 \%$ des naissances et réalisent une part équivalente des FIV (62\%).

(2) Centre d'étude et de conservation des œufs et du sperme humains
La situation est très différente concernant l'AMP avec tiers donneur. La France ne réalise que $2 \%$ des dons d'ovocytes de l'UE. L'Allemagne et l'Italie n'en réalisent aucun puisque ces pays les interdisent. Ces trois poids lourds démographiques, qui comprennent à eux trois $39 \%$ de la population féminine âgée de 20 à 44 ans de l'UE, sont donc très en retrait pour ce qui est du don d'ovocytes. Leurs ressortissants doivent franchir les frontières pour pouvoir réaliser leurs projets parentaux [5], entraînant un phénomène de recours transfrontalier qui reste à quantifier. Les pays de destination sont l'Espagne, mais également des pays comme la Grèce et la République tchèque, qui ne représentent chacun que $2 \%$ des naissances de l'UE. Ces trois pays réalisent à eux seuls $77 \%$ de l'activité de don d'ovocytes de l'UE : $59 \%$ pour l'Espagne, qui domine le paysage, $9 \%$ pour la République tchèque, et $8 \%$ pour la Grèce.

Le déséquilibre est marqué également pour le don de sperme. Il touche notamment là encore l'Allemagne, l'Italie et la France. En France, comme déjà mentionné, l'accès au don de sperme n'est autorisé qu'aux couples hétérosexuels, entraînant un recours transfrontalier des « exclus » de l'AMP, couples de femmes et femmes seules en particulier. La situation est identique en Allemagne avec une activité de don de sperme mal connue, les statistiques pour ce pays ne figurant pas dans le bilan européen (encadré 2). En Italie, le don de sperme est interdit. Au sein de l'UE, le don de sperme est essentiellement réalisé dans trois pays : deux pays, le Danemark et la Belgique, qui ne représentent respectivement que $1 \%$ et $2 \%$ des naissances de l'UE, mais assurent $26 \%$ et $20 \%$ des dons de sperme de l'UE, et l'Espagne, qui représente $8 \%$ des naissances et assure $19 \%$ des dons de sperme.

Au sein de l'Union européenne, l'AMP avec tiers donneur se concentre donc dans quelques pays qui accueillent les Européens qui ne peuvent accéder au don de gamètes chez eux. Cette très forte concentration du don dans quelques pays, souvent de petite taille, soulève des questions éthiques sur son organisation. Pour répondre à la demande de la population européenne, ces pays doivent recruter un nombre important de donneurs qui ne peut reposer sur le seul altruisme de la population locale. Les donneuses pourraient alors en partie être des jeunes femmes en situation de fragilité économique, motivées par la compensation financière de $400 €$ à $2000 €$ versée par les centres [6].

Des États généraux de la bioéthique ontétélancés en France en janvier 2018, appelant la société à réfléchir, entre autres, à de possibles évolutions du cadre législatif del'AMP. L'accès à l'AMP pour toutes les femmes, y compris celles vivant seules ou en couple de même sexe, est débattu et pourrait conduire à une révision de la loi de bioéthique dans l'année. Les choix qui seront faits auront des conséquences au-delà de nos frontières, accentuant ou freinant le mouvement de concentration de l'activité européenne d'AMP avec don de gamètes dans quelques pays. 


\section{Encadré 2. Les sources d'information}

\section{POUR LA FRANCE}

Période 1981-1999 : bilans d'activité de FIV réalisés périodiquement par le Groupement de l'étude des FIV en France (GEFF) puis par le ministère de la Santé. Ces données sont complétées par celles du registre FIVNAT. Un bilan de ces données est accessible dans le tableau 2 de la référence bibliographique [1].

NB : les données ne sont pas disponibles pour 2000 et 2001; pour ces années, le nombre d'enfants a été estimé sous I'hypothèse d'une tendance linéaire.

Période 2002-2015 : bilans d'activité de l'AMP publiés annuellement par l'Agence de la Biomédecine et portant sur l'activité FIV et IA.

Accès libre aux données: https://www.agence-biomedecine. fr/AMP

Période 2016-2019 : projection des tendances observées sous I'hypothèse d'évolution linéaire.

Pour toutes les années : les statistiques de naissances totales issues de l'état civil et publiées par l'Insee.

Accès libre : https://www.insee.fr

\section{POUR L'EUROPE}

Les statistiques d'activité de FIV en Europe publiées régulièrement par la Société européenne de reproduction humaine et d'embryologie [2]. Ce bilan n'est pas restreint à I'Union européenne, et il inclut des pays tels que la Russie. L'analyse présentée ici a été limitée aux 28 pays de l'Union européenne parmi lesquels 26 ont participé au bilan publié en 2017 sur l'activité d'AMP de l'année 2013 (exceptés le Luxembourg et la Slovaquie). Au sein de ces 26 pays, 1083 centres de FIV fonctionnent et l'activité de 957 d'entre eux (88 \%) a été incluse dans le bilan. La France, l'Italie, l'Allemagne et le Royaume-Uni cumulent à eux quatre 513 centres dont 512 ont fourni des données. En Espagne, seuls 130 centres parmi les 198 du pays l'ont fait. Les estimations présentées ici ont été redressées en faisant l'hypothèse que les centres de FIV inclus dans le bilan étaient représentatifs de l'ensemble de l'activité du pays. Le don d'ovocytes est inclus dans l'activité FIV, et le don de spermatozoïdes en termes d'insémination artificielle (IAD), mais l'information sur les dons sperme en FIV n'est pas présentée. Certains pays ne transmettent pas de données concernant I'IAD. Dans l'Union européenne, cela est le cas en particulier de l'Allemagne et de la République tchèque.

Les statistiques démographiques (natalité, femmes en âge reproductif) sont publiées par Eurostat.

Accès libre : http://ec.europa.eu/eurostat

\section{Références}

[1] de La Rochebrochard E., 2003, « Des hommes médicalement assistés pour procréer: IAD, FIV, ICSI, bilan d'une révolution dans la prise en charge médicale de l'infertilité masculine », Population, 58(45), p. 549-586 [https://www.persee.fr/doc/pop_0032-4663_2003_ num_58_4_7413].

[2] Calhaz-Jorge C. et al., 2017, « Assisted reproductive technology in Europe, 2013: Results generated from European registers by ESHRE », Human Reproduction, 32(10), p. 1957-1973 [https://academic.oup. com/humrep/article/32/10/1957/4096426].

[3] Adamson G.D., Tabangin M., Macaluso M., de Mouzon J., 2013, «The number of babies born globally after treatment with the assisted reproductive technologies », Fertility and Sterility, 100(Suppl 3), p. S42 [http://www.fertstert.org/article/S0015-0282(13)02586-7/pdf ].

[4] Rozée Gomez V., de La Rochebrochard E., 2013, « Cross-border reproductive care among French patients: Experiences in Greece, Spain and Belgium », Human Reproduction, 28(11), p. 3103-3110 [https://www.ncbi.nlm.nih.gov/pmc/articles/PMC3795470/pdf/ det326.pdf].

[5] Shenfield F., de Mouzon J., Pennings G., Ferraretti A.P., Andersen A.N., De Wert G., Goossens V., 2010, "Cross border reproductive care in six European countries », Human Reproduction, 25(6), p. 1361-1368 [https://academic.oup.com/humrep/article/25/6/1361/2915759].

[6] Pennings G., de Mouzon J., Shenfield F., Ferraretti A.P., Mardesic T., Ruiz A., Goossens V., 2014, « Socio-demographic and fertility-related characteristics and motivations of oocyte donors in eleven European countries », Human Reproduction, 29(5), p. 1076-1089 [https:// academic.oup.com/humrep/article/29/5/1076/675905].

\section{Résumé}

En France, un enfant sur 30 est désormais conçu grâce à une technique d'assistance médicale à la procréation (AMP), qu'il s'agisse d'une fécondation in vitro (FIV) ou d'une insémination artificielle (IA). La FIV est à elle seule à l'origine de la naissance d'un enfant sur 40. Cette proportion croît de manière linéaire depuis plus de trois décennies. Globalement, 300000 enfants ont été conçus par FIV entre 1981 et fin 2014. Si la tendance se poursuit, un total de 400000 sera atteint fin 2019. Seul $5 \%$ de l'activité d'AMP française est réalisée avec un don de gamètes, en général de spermatozoïde, les dons d'ovocytes connaissant une pénurie importante. La législation française restreint l'accès de l'AMP aux couples hétérosexuels d'âge reproductif. Dans ce contexte législatif et organisationnel, des Français vont à l'étranger pour pouvoir accéder à une AMP avec don de gamètes, ils se tournent vers l'Espagne, la Grèce, la République tchèque, la Belgique ou le Danemark.

\section{Mots-clés}

Assistance médicale à la procréation, fécondation in vitro, AMP transnationale, naissances, France, Europe.
Ined : 133, boulevard Davout, 75980 Paris, Cedex 20 Directrice de la publication : Magda Tomasini Rédacteur en chef : Gilles Pison Éditrice : Marie-Paule Reydet Graphiste : Isabelle Milan

Impression : Mérico Delta Print, Bozouls, France D. L. 2e trim. $2018 \cdot$ ISSN 01847783
Retrouvez Population et Sociétés

dès sa parution sur le site internet de l'Ined et abonnez-vous :

www.ined.fr/fr/publications/populationet-societes

Contact : edition@ined.fr

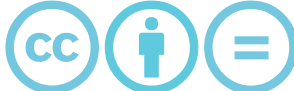

Cet article peut être reproduit sur papier ou en ligne gratuitement en utilisant notre licence Creative Commons 